\title{
ON THE SOLUTIONS OF FALKNER-SKAN EQUATION
}

\author{
LABBAOUI FATMA ${ }^{1,2, *}$, AND AIBOUDI MOHAMMED ${ }^{1,2,}$ \\ ${ }^{1}$ Département de Mathématique, Faculté des Sciences Exactes et Appliquées, \\ Université Oran 1 Ahmed Ben Bella, Oran, Algérie \\ ${ }^{2}$ Laboratoire de recherche d'Analyse Mathématique et Application L.A.M.A, Oran, Algerie \\ *Corresponding author: fatimazahra107@yahoo.fr
}

\begin{abstract}
We consider the differential equation $f^{\prime \prime \prime}+f f^{\prime \prime}+\beta\left(f^{\prime 2}-1\right)=0$, with $\beta>0$. In order to prove the existence of solutions satisfying the boundary conditions $f(0)=a \geq 0, f^{\prime}(0)=b \geq 0$ and $f^{\prime}(+\infty)=-1$ or 1 for $0<\beta \leq \frac{1}{2}$. We use shooting technique and consider the initial conditions $f(0)=a, f^{\prime}(0)=b$ and $f^{\prime \prime}(0)=c$. We prove that there exists an infinitely many solutions such that $f^{\prime}(+\infty)=1$.
\end{abstract}

\section{INTRODUCTION}

In 1931 the Falkner-Skan equation is introduced for studying the boundary layer flow past a semi infinite wedge, it is defined by

$$
f^{\prime \prime \prime}+f f^{\prime \prime}+\beta\left(f^{\prime 2}-1\right)=0
$$

The solution of this equation have been studied by numerous authors as, for example, D. R. Hartree (1937), H. Weyl (1942), W. A. Coppel (1960), P. Hartman (1964) and G.C. Yang (2003,2004).

The more general equation of (1.1) is

$$
f^{\prime \prime \prime}+f f^{\prime \prime}+g\left(f^{\prime}\right)=0
$$

where $g: \mathbb{R} \longrightarrow \mathbb{R}$ is some function. The solutions obtained are called similarity solutions.

Received February $12^{\text {th }}, 2020$; accepted March $9^{\text {th }}, 2020$; published May $1^{\text {st }}, 2020$.

2010 Mathematics Subject Classification. 76D10, 34B15.

Key words and phrases. boundary layer; shooting technique; convex solution; concave solution; convex-concave solution. (C)2020 Authors retain the copyrights of their papers, and all open access articles are distributed under the terms of the Creative Commons Attribution License. 
The most famous example is perhaps the Blasius equation (1908), which corresponds to $g(x)=0$ and arises in the study of laminar boundary layer on a flat plate.

More recently, the equation (1.2) with $g(x)=\beta x^{2}$ and $g(x)=\beta x(x-1)$ has been considered. These cases occur, for example, in the study of free convection and of mixed convection boundary layer flows over a vertical surface embedded in a porous medium.

Most of the time, associated with the equation (1.1) is the boundary value problem:

$$
\left\{\begin{array}{l}
f^{\prime \prime \prime}+f f^{\prime \prime}+\beta\left(f^{\prime 2}-1\right)=0 \\
f(0)=a \\
f^{\prime}(0)=b \\
f^{\prime}(t) \longrightarrow \lambda \text { as } t \longrightarrow+\infty
\end{array}\right.
$$

To solve the boundary value problem $\left(\mathcal{P}_{\beta ; a, b, \lambda}\right)$ we will use the shooting technique. To this end, let $f_{c}$ denote the solution of the initial value problem $\left(\mathcal{Q}_{\beta} ; a, b, c\right)$ consisting in the equation (1.1) together with

the initial conditions $f_{c}(0)=a, f_{c}^{\prime}(0)=b$ and $f_{c}^{\prime \prime}(0)=c$, and let $\left[0, T_{c}\right.$ [ be the right maximal interval of existence of $f_{c}$. To obtain a solution of $\left(\mathcal{P}_{\beta} ; a, b, \lambda\right)$ amounts to find a value of $c$ such that $T_{c}=+\infty$ and $f_{c}^{\prime}(t) \longrightarrow \lambda$ as $t \longrightarrow+\infty$.

We must assume that $\beta\left(\lambda^{2}-1\right)=0$ to have solutions, in our case of Falkner-Skan equation, the only relevant conditions are $f_{c}^{\prime}(t) \longrightarrow-1$ or $f_{c}^{\prime}(t) \longrightarrow 1$ as $t \longrightarrow+\infty$.

In the following, we will study the existence of concave, convex, concave-convex and convex-concave solutions to the boundary value problem $\left(\mathcal{P}_{\beta ; a, b,-1}\right)$ and $\left(\mathcal{P}_{\beta ; a, b, 1}\right)$ for $0<\beta \leq \frac{1}{2}, a \geq 0$ and $b \geq 0$.

\section{Preliminary Results}

Let $f$ be a solution of the equation (1.1) on some interval $I$, we consider the function $H_{f}: I \longrightarrow \mathbb{R}$ defined by

$$
H_{f}=f^{\prime \prime}+f\left(f^{\prime}-1\right)
$$

This function is obtained by integrating the equation (1.1), in fact, if $f$ is a solution of (1.1) then

$$
H_{f}^{\prime}=\left(f^{\prime}-1\right)\left((1-\beta) f^{\prime}-\beta\right)
$$

We give lemmas that will be useful later.

Lemma 2.1. Let $f$ be a solution of (1.1) on some maximal interval $I$. If there exists $t_{0} \in I$ such that $f^{\prime}\left(t_{0}\right) \in\{-1,1\}$ and $f^{\prime \prime}\left(t_{0}\right)=0$, then $I=\mathbb{R}$ and $f^{\prime \prime}(t)=0$ for all $t \in \mathbb{R}$.

Proof. See [4], proposition 3.1 item 3.

Lemma 2.2. Let $\beta>0$ and $f$ be a solution of equation (1.1) on some interval $I$, such that $f^{\prime}$ is not constant. 
(1) If there exists $s<r \in I$ such that $f^{\prime \prime}(s) \leq 0$ and $\left(f^{\prime 2}-1\right)>0$ on $] s, r\left[\right.$, then $f^{\prime \prime}(t)<0$ for all $t \in] s, r]$.

(2) If there exists $s<r \in I$ such that $f^{\prime \prime}(s) \geq 0$ and $\left(f^{\prime 2}-1\right)<0$ on $] s, r\left[\right.$, then $f^{\prime \prime}(t)>0$ for all $t \in] s, r]$.

(3) If there exists $s<r \in I$ such that $f^{\prime \prime}<0$ on $] s, r\left[\right.$ and $f^{\prime \prime}(r)=0$, then $\left(f^{\prime 2}(r)-1\right)<0$.

(4) If there exists $s<r \in I$ such that $f^{\prime \prime}>0$ on $] s, r\left[\right.$ and $f^{\prime \prime}(r)=0$, then $\left(f^{\prime 2}(r)-1\right)>0$.

Proof. Let $F$ denote any primitive function of $f$. From (1.1) we deduce the relation

$$
\left(f^{\prime \prime} \exp F\right)^{\prime}=-\beta\left(f^{\prime 2}-1\right) \exp F
$$

All the assertions 1-4 follow easily from this relation and from previous lemma. Let us verify the first and the third of these assertions. For the first one, since $\psi=f^{\prime \prime} \exp F$ is decreasing on $[s, r]$, we obtain

$$
\begin{aligned}
t \geq s & \Longrightarrow \psi(t) \leq \psi(s) \\
& \Longrightarrow f^{\prime \prime}(t) \exp F(t) \leq f^{\prime \prime}(s) \exp F(s) \\
& \Longrightarrow f^{\prime \prime}(t) \leq f^{\prime \prime}(s) \exp (F(s)-F(t)) \\
& \left.\left.\Longrightarrow f^{\prime \prime}(t) \leq 0, \forall t \in\right] s, r\right]
\end{aligned}
$$

For the third one, since $\psi<0$ on $] s, r\left[\right.$ and $\psi(r)=0, \psi^{\prime}(t) \geq 0$ on $[s, r]$, then $\psi^{\prime}(r) \geq 0$.

$$
\psi^{\prime}(r)=-\beta\left(f^{\prime 2}(r)-1\right) \exp F \geq 0
$$

This and lemma 2.1 imply that $\left(f^{\prime 2}(r)-1\right)<0$.

Lemma 2.3. Let $f$ be a solution of (1.1) on some maximal interval $] T_{-}, T_{+}\left[\right.$. If $T_{+}$is finite, then $f^{\prime}$ and $f^{\prime \prime}$ are unbounded in any neighborhood of $T_{+}$.

Proof. See [4], proposition 3.1 item 6.

Lemma 2.4. Let $\beta \neq 0$. If $f$ is a solution of (1.1)on some interval $] \tau,+\infty\left[\right.$ such that $f^{\prime}(t) \longrightarrow \lambda$ as $t \longrightarrow+\infty$, then $\lambda \in\{-1,1\}$. Moreover, if $f$ is of constant sign at infinity, then $f^{\prime \prime}(t) \longrightarrow 0$ as $t \longrightarrow+\infty$.

Proof. See [4], proposition 3.1 item 4 and 5.

Lemma 2.5. Let $\beta>0$ and $f$ be a solution of equation (1.1) on some right maximal interval $I=[\tau,+\infty[$. If $f \geq 0$ and $f^{\prime} \geq 0$ on $I$, then $T_{+}=+\infty$ and $f^{\prime}$ is bounded on $I$. 
Proof. Let $L=L_{f}$ be the function defined on $I$ by

$$
L(t)=3 f^{\prime \prime^{2}}(t)+\beta f^{\prime}(t)\left(2{f^{\prime 2}}^{2}(t)-6\right) .
$$

Easily, using (1.1), we obtain that

$$
L^{\prime}(t)=-6 f(t) f^{\prime \prime^{2}}(t) \quad \forall t \in I
$$

and, since $f \geq 0$ on $I$ this implies that $L$ is decreasing. Hence

$$
\begin{gathered}
\forall t \in I=\left[\tau, T_{+}[: t>\tau \Longrightarrow L(t) \leq L(\tau)\right. \\
\beta f^{\prime}(t)\left(2{f^{\prime}}^{2}(t)-6\right) \leq 3 f^{\prime^{2}}(t)+\beta f^{\prime}(t)\left(2 f^{\prime^{2}}(t)-6\right) \leq L(\tau), \forall t \in I .
\end{gathered}
$$

It follows that $f^{\prime}$ is bounded on $I$ and thanks to lemma (2.3) that $T_{+}=+\infty$.

Lemma 2.6. Let $\beta>0$ and $f$ be a solution of equation (1.1) on some right maximal interval $I=\left[\tau, T_{+}[\right.$. If $f(\tau) \geq 0, f^{\prime}(\tau) \geq 1 \quad$ and $f^{\prime \prime}(\tau)>0$, then there exists $\left.t_{0} \in\right] \tau, T_{+}\left[\right.$such that $f^{\prime \prime}>0$ on $\left[\tau, t_{0}[\right.$ and $f^{\prime \prime}\left(t_{0}\right)=0$.

Proof. Assume for contradiction that $f^{\prime \prime}>0$ on $I$. Then $f(t) \geq 0, f^{\prime}(t) \geq 1$ for all $t \in I$. Then, we have

$$
f^{\prime \prime \prime}=-f f^{\prime \prime}-\beta\left(f^{\prime 2}-1\right) \leq 0
$$

It follows that $0<f^{\prime \prime}(t) \leq c$ for all $t \in I$ and hence, by lemma (2.3), we have $T_{+}=+\infty$.

Next, let $s>\tau$ and $\epsilon=\beta\left(f^{\prime}(s)^{2}-1\right)$. One has $\epsilon>0$ and, comming back to (2.3), we obtain

$$
f^{\prime \prime \prime} \leq-\epsilon \text { on }[s,+\infty[
$$

After integrating, we get

$$
\forall t \geq s, f^{\prime \prime}(t)-f^{\prime \prime}(s) \leq-\epsilon(t-s)
$$

and a contradiction with the fact that $f^{\prime \prime}(t)>0$. Consequently, there exists $\left.t_{0} \in\right] \tau, T_{+}\left[\right.$such that $f^{\prime \prime}>0$ on $\left[\tau, t_{0}\left[\right.\right.$ and $f^{\prime \prime}\left(t_{0}\right)=0$.

Lemma 2.7. Let $\beta \in] 0, \frac{1}{2}[$ and $f$ be a solution of equation (1.1) on some right maximal interval $I=$ ]$T_{-}, T_{+}\left[\right.$. If there exists $t_{0} \in I$ such that $\frac{\beta}{1-\beta}<f^{\prime}\left(t_{0}\right)<1$ and $0 \leq f^{\prime \prime}\left(t_{0}\right) \leq f\left(t_{0}\right)\left(1-f^{\prime}\left(t_{0}\right)\right)$, then $T_{+}=+\infty$ and $f^{\prime}(t) \longrightarrow 1$ as $t \longrightarrow+\infty$. Moreover $f^{\prime \prime}>0$ on $\left[t_{0},+\infty[\right.$. 
Proof. Let $\delta=\sup X\left(t_{0}\right)$, where

$$
X\left(t_{0}\right)=\{t \in] t_{0}, T_{+}\left[: f^{\prime}\left(t_{0}\right)<f^{\prime}<1 \text { and } f^{\prime \prime}>0 \text { on }\right] t_{0}, t[\} .
$$

The set $X\left(t_{0}\right)$ is not empty. This is clear if $f^{\prime \prime}\left(t_{0}\right)>0$, and if $f^{\prime \prime}\left(t_{0}\right)=0$ it follows from the fact that

$$
f^{\prime \prime \prime}\left(t_{0}\right)=-\beta\left(f^{\prime 2}\left(t_{0}\right)-1\right)>0 .
$$

We claim that $\delta=T_{+}$, assume for contradiction that $\delta<T_{+}$. From lemma (2.2), item 2, we get that $f^{\prime \prime}(\delta)>0$, which implies, by definition of $\delta$, that $f^{\prime}(\delta)=1$. Therefore, since the function $H_{f}$ defined by $(2.1)$ is nonincreasing on $\left[t_{0}, \delta\right]$, we obtain

$$
\begin{aligned}
\delta \geq t_{0} & \Longrightarrow H_{f}(\delta) \leq H_{f}\left(t_{0}\right) \\
& \Longrightarrow f^{\prime \prime}(\delta) \leq f^{\prime \prime}\left(t_{0}\right)-f\left(t_{0}\right)\left(1-f^{\prime}\left(t_{0}\right)\right) \\
& \Longrightarrow f^{\prime \prime}(\delta)<0
\end{aligned}
$$

a contradiction. Thus, we have $\delta=T_{+}$. From lemma 2.3, it follows that $T_{+}=+\infty$. Since $f^{\prime \prime}>0$ on $\left[t_{0},+\infty\left[\right.\right.$, by virtue of lemma 2.4 , we get that $f^{\prime}(t) \longrightarrow 1$ as $t \longrightarrow+\infty$.

Lemma 2.8. Let $\left.\beta \in] 0, \frac{1}{2}\right]$ and $f$ be a solution of equation (1.1) on some right maximal interval $I=$ ]$T_{-}, T_{+}\left[\right.$. If there exists $t_{0} \in I$ such that $f^{\prime}\left(t_{0}\right)>1$ and $f\left(t_{0}\right)\left(1-f^{\prime}\left(t_{0}\right)\right) \leq f^{\prime \prime}\left(t_{0}\right) \leq 0$. Then $T_{+}=+\infty$ and $f^{\prime}(t) \longrightarrow 1$ as $t \longrightarrow+\infty$. Moreover $f^{\prime \prime}<0$ on $\left[t_{0},+\infty[\right.$.

Proof. If we set $\omega=\sup Y\left(t_{0}\right)$, where

$$
Y\left(t_{0}\right)=\{t \in] t_{0}, T_{+}\left[: 1<f^{\prime}<f^{\prime}\left(t_{0}\right) \text { and } f^{\prime \prime}<0 \text { on }\right] t_{0}, t[\} .
$$

The conclusion will follow by proceeding in the same way as the previous proof.

\section{Description of our APproach When $b \geq 1$}

Let $\beta>0, a \geq 0$ and $b \geq 1$. As said in the introduction, the method we will use to obtain solutions of the boundary value problems $\left(\mathcal{P}_{\beta ; a, b,-1}\right)$ and $\left(\mathcal{P}_{\beta ; a, b, 1}\right)$ is the shooting technique. Specifically, for $c \in \mathbb{R}$, let us denote by $f_{c}$ the solution of equation (1.1) satisfying the initial conditions

$$
f_{c}(0)=a, f_{c}^{\prime}(0)=b \text { and } f_{c}^{\prime \prime}(0)=c
$$

and let $\left[0, T_{c}\right.$ [ be the right maximal interval of existence of $f_{c}$. Hence, finding a solution of one of the problems $\left(\mathcal{P}_{\beta ; a, b,-1}\right)$ and $\left(\mathcal{P}_{\beta ; a, b, 1}\right)$ amounts to finding a value of $c$ such that $T_{+}=+\infty$ and $f_{c}^{\prime}(t) \longrightarrow-1$ or 1 as $t \longrightarrow+\infty$. 
To this end, let us partition $\mathbb{R}$ into the four sets $C_{0}, C_{1}, C_{2}$ and $C_{3}$ defined as follows.

Let $\left.C_{0}=\right] 0,+\infty[$ and, according to the notations used in [4], let us set

$$
\begin{gathered}
C_{1}=\left\{c \leq 0 ; 1 \leq f _ { c } ^ { \prime } \leq b \text { and } f _ { c } ^ { \prime \prime } \leq 0 \text { on } \left[0, T_{c}[\},\right.\right. \\
C_{2}=\left\{\begin{array}{c}
c \leq 0 ; \exists t_{c} \in\left[0, T_{c}\left[, \quad \exists \epsilon_{c}>0 \text { such that } f_{c}^{\prime}>1 \text { on }\right] 0, t_{c}[,\right. \\
\left.f_{c}^{\prime}<1 \text { on }\right] t_{c}, t_{c}+\epsilon_{c}\left[\text { and } f _ { c } ^ { \prime \prime } \leq 0 \text { on } \left[0, t_{c}+\epsilon_{c}[\right.\right.
\end{array}\right\}, \\
C_{3}=\left\{\begin{array}{c}
c \leq 0 ; \exists r_{c} \in\left[0, T_{c}\left[, \quad \exists \eta_{c}>0 \text { such that } f_{c}^{\prime \prime}<0 \text { on }\right] 0, r_{c}[\right. \\
\left.f_{c}^{\prime \prime}>0 \text { on }\right] r_{c}, \quad r_{c}+\eta_{c}\left[\text { and } f_{c}^{\prime}>1 \text { on }\right] 0, r_{c}+\eta_{c}[
\end{array}\right\} .
\end{gathered}
$$

This is obvious that $C_{0}, C_{1}, C_{2}$ and $C_{3}$ are disjoint sets and that their union is the whole line of real numbers. Thanks to lemma 2.3 and 2.4 if $c \in C_{1}$ then $T_{+}=+\infty$ and $f_{c}^{\prime}(t) \longrightarrow 1$ as $t \longrightarrow+\infty$. In fact, $C_{1}$ is the set of values of $c$ for which $f_{c}$ is a concave solution of $\left(\mathcal{P}_{\beta ; a, b, 1}\right)$. Since $\beta>0$, the study done in [4] (specially in section 5.2) says, on the one hand, that $C_{3}=\varnothing$ (which can easily be deduced from lemma 2 , item 1) and, on the other hand, that either $C_{1}=\varnothing$ and $\left.\left.C_{2}=\right]-\infty, 0\right]$, or there exist $c^{*} \leq 0$ such that $C_{1}=\left[c^{*}, 0\right]$ and $\left.C_{2}=\right]-\infty, c^{*}[$.

In addition, from the lemma 5.16 in [4], if $\left.\beta \in] 0, \frac{1}{2}\right]$ then we are in the second case and $c^{*} \leq-a(b-1)$.

In order to complete the study, let us divide the set $C_{2}$ into the following two subsets

$$
\begin{gathered}
C_{2,1}=\left\{c \in C _ { 2 } : f _ { c } ^ { \prime } > - 1 \text { on } \left[0, T_{c}[\},\right.\right. \\
C_{2,2}=\left\{c \in C_{2}: \exists s_{c} \in\right] 0, T_{c}\left[\text { such that } f _ { c } ^ { \prime } > - 1 \text { on } \left[0, s_{c}\left[\text { and } f_{c}^{\prime}\left(s_{c}\right)=-1\right\} .\right.\right.
\end{gathered}
$$

And let us give properties of each of them that hold for all $\left.\beta \in] 0, \frac{1}{2}\right]$.

Lemma 3.1. If $c \in \mathbb{R}$ such that $f_{c}^{\prime}>0$ on $\left[0, T_{c}\left[\right.\right.$, then $T_{c}=+\infty$ and $f_{c}^{\prime}$ is bounded. Moreover, if $c \leq 0$, then $f_{c}^{\prime} \leq \max \{b, \sqrt{3}\}$ on $[0,+\infty[$.

Proof. Let $c \in \mathbb{R}$ is such that $f_{c}^{\prime}>0$ on $\left[0, T_{c}\left[\right.\right.$, then $f_{c} \geq a \geq 0$ on $\left[0, T_{c}[\right.$ and thanks to lemma 2.5 , it follows that $T_{c}=+\infty$ and $f_{c}^{\prime}$ is bounded.

It remains to show that $f_{c}^{\prime} \leq \max \{b, \sqrt{3}\}$ in the case where $c \leq 0$. As in [4], let us define the function $L_{c}$ on $[0,+\infty[$ by

$$
L_{c}(t)=3 f_{c}^{\prime^{2}}(t)+\beta f_{c}^{\prime}(t)\left(2{f_{c}^{\prime 2}}^{2}(t)-6\right)
$$

and since $f_{c} \geq 0$, it implies that $L_{c}$ is decreasing. 
If $f_{c}^{\prime \prime} \leq 0$ on $] 0,+\infty\left[\right.$, then $f_{c}^{\prime} \leq b$. Otherwise, there exists $t_{0}$ such that $f_{c}^{\prime \prime}<0$ on $] 0, t_{0}\left[\right.$ and $f_{c}^{\prime \prime}\left(t_{0}\right)=0$. By lemma 2.2 item 3 , it follows that $f_{c}^{\prime}<1$, and thus $L_{c}\left(t_{0}\right)<0$. Then, $L_{c}<0$ on $] t_{0},+\infty[$ which implies that $f_{c}^{\prime} \leq \sqrt{3}$ on $] t_{0},+\infty\left[\right.$. Since $f_{c}^{\prime} \leq b$ on $] 0, t_{0}[$, the proof is complete.

Proposition 3.1. Let $c_{*}=\inf \left(C_{1} \cup C_{2,1}\right)$. Then $c_{*}$ is finite.

Proof. let $c \in C_{1} \cup C_{2,1}$. By definition of $C_{1}$ and $C_{2,1}$, and thanks to lemma 2.3, we have $T_{c}=+\infty$ and $0<$ $f_{c}^{\prime}<d$ on $[0,+\infty[$ where $d=\max \{b, \sqrt{3}\}$. Since

$$
\begin{aligned}
\left(f_{c}^{\prime \prime}+f_{c} f_{c}^{\prime}\right)^{\prime} & =f_{c}^{\prime \prime \prime}+f_{c} f_{c}^{\prime \prime}+{f_{c}^{\prime 2}}^{2} \\
& =-\beta\left({f_{c}^{\prime 2}}^{2}-1\right)+{f_{c}^{\prime 2}}^{2} \\
& =-\beta{f_{c}^{\prime 2}}^{2}+\beta+{f_{c}^{\prime 2}}^{\prime 2} \beta+d^{2} .
\end{aligned}
$$

By integrating, we then have

$$
\forall t \geq 0, \quad f_{c}^{\prime \prime}(t)+f_{c}(t) f_{c}^{\prime}(t) \leq c+a b+\left(\beta+d^{2}\right) t .
$$

Integrating once again, for all $t \geq 0$, we get

$$
0<f_{c}^{\prime}(t) \leq f_{c}^{\prime}(t)+\frac{1}{2} f_{c}^{2}(t) \leq b+\frac{1}{2} a^{2}+(c+a b)+\frac{1}{2}\left(\beta+d^{2}\right) t^{2} .
$$

Which implies that

$$
c \geq-a b-\sqrt{\left(2 b+a^{2}\right)\left(\beta+d^{2}\right)} .
$$

Remark 3.1. As we have seen above, if $C_{1} \neq \varnothing$, then $C_{1}=\left[c^{*}, 0\right]$ and thus $C_{2,1} \subset\left[c_{*}, c^{*}[\right.$.

$$
\text { 4. The CASE } \left.\beta \in] 0, \frac{1}{2}\right] \text { AND } b \geqslant 1
$$

In this section we assume that $\left.\beta \in] 0, \frac{1}{2}\right], a \geqslant 0$ and $b \geqslant 1$.

Proposition 4.1. If $c>0$, then $T_{c}=+\infty$ and $f_{c}^{\prime}(t) \rightarrow 1$ as $t \rightarrow+\infty$.

Proof. From lemma 2.6, there exists $\left.t_{0} \in\right] 0, T_{c}\left[\right.$ such that $f_{c}^{\prime \prime}>0$ on $\left[0, t_{0}\left[\right.\right.$ and $f_{c}^{\prime \prime}\left(t_{0}\right)=0$. Since $f_{c}\left(t_{0}\right)>0$ and $f_{c}^{\prime}\left(t_{0}\right)>b>1$. Thus $f_{c}\left(t_{0}\right)\left(1-f_{c}^{\prime}\left(t_{0}\right)\right) \leqslant f_{c}^{\prime \prime}\left(t_{0}\right)=0$, the conclusion follows from lemma 2.8 .

Remark 4.1. Thanks to the previous proposition, we see that $f_{c}$ is a convex-concave solution of $\left(\mathcal{P}_{\beta ; a}, b, 1\right)$ for all $c>0$.

Proposition 4.2. There exists $c^{*} \leq-a(b-1)$ such that $C_{1}=\left[c^{*}, 0\right]$. 
Proof. If $b=1$ then $C_{1}=\{0\}$.

If $b>1$, as we already said in the previous section, this result is proven in [4] (see corollary 5.13 and lemma $5.16)$, let us recall briefly the main arguments which where used to get it. On the one hand, from lemma 2.8 with $t_{0}=0$ (or lemma 5.16 of [4]), it follows that $[-a(b-1), 0] \subset C_{1}$. On the other hand, lemma 5.12 of [4] implies that $C_{2}$ is an interval of the type $]-\infty, c^{*}\left[\right.$. This complete the proof since $\left.\left.C_{1}=\right]-\infty, 0\right] \backslash C_{2}$.

Remark 4.2. From the previous proposition, we have that $0 \notin C_{2.2}$.

Proposition 4.3. If $c \in C_{2.1}$, then $T_{c}=+\infty$ and $f_{c}^{\prime}$ has a finite limit at infinity, equal either to -1 or to 1 .

Proof. Let $c \in C_{2.1}$. By proposition 4.2, we have $c<0$.

Assume first that $f_{c}^{\prime \prime}<0$ on $] 0,+\infty\left[\right.$. Then $f_{c}^{\prime}$ is decreasing, and thus $f_{c}^{\prime}$ has a finite limit $\lambda$ at infinity. Moreover, by definition of the set $C_{2.1}$ we get

$$
\exists t_{c} \in\left[0,+\infty\left[\text { such that } f_{c}^{\prime}\left(t_{c}\right)=1\right.\right.
$$

and by lemma 2.4, we finally get that $\lambda=-1$.

Assume now that $f_{c}^{\prime \prime}$ vanishes on $] 0,+\infty\left[\right.$, let $t_{0}$ be the first point where $f_{c}^{\prime \prime}$ vanishes. Thanks to lemma 2.2 item 3 , we have $0<f_{c}^{\prime}\left(t_{0}\right)<1$, and the conclusion follows from lemma $2.7(\lambda=1)$.

Remark 4.3. If $c \in C_{2.1}$ then either $f_{c}$ is a concave solution of $\left(\mathcal{P}_{\beta ; a, b,-1}\right)$ or $f_{c}$ is a concave-convex solution of $\left(\mathcal{P}_{\beta} ; a, b, 1\right)$.

Proposition 4.4. Let $c$ be a point of the boundary of $C_{2.2}$. Then $c \in C_{2.1}$ and $f_{c}^{\prime}(t) \longrightarrow-1$ as $t \longrightarrow+\infty$.

Proof. See [3] in the case of the mixed convection equation.

Proposition 4.5. There exists at most one $c$ such that $f_{c}^{\prime}(t) \longrightarrow-1$ as $t \longrightarrow+\infty$.

Proof. From proposition 4.2 and 4.3 , we see that if $c$ is such that $f_{c}^{\prime}(t) \longrightarrow-1$ as $t \longrightarrow+\infty$, then $c<0$ and $f_{c}^{\prime \prime}<0$. By the change of variable, as done in [4], section 4 , we can define a function $\left.\left.v:\right] 0, b^{2}\right] \rightarrow \mathbb{R}$ such that

$$
\forall t \geq 0, \quad v\left(f_{c}^{\prime^{2}}(t)\right)=f_{c}(t)
$$

By setting $y=f_{c}^{\prime^{2}}(t)$, we get

$$
f_{c}(t)=v(y), \quad f_{c}^{\prime}(t)=\sqrt{y}, \quad f_{c}^{\prime \prime}(t)=\frac{1}{2 v^{\prime}(y)} \quad \text { and } \quad f_{c}^{\prime \prime \prime}(t)=-\frac{v^{\prime \prime}(y) \sqrt{y}}{2 v^{\prime 3}(y)}
$$


and using (1.1) we obtain

$$
\left.\forall y \in] 0, b^{2}\right], \quad v^{\prime \prime}(y)=\frac{v(y) v^{\prime^{2}}(y)}{\sqrt{y}}+\frac{2 \beta(y-1)}{\sqrt{y}} v^{\prime^{3}}(y)^{3} .
$$

From (3.1), we deduce that $v\left(b^{2}\right)=a$ and $v^{\prime}\left(b^{2}\right)=\frac{1}{2 c}$. Moreover, since $f_{c}$ is bounded, it is so for $v$.

Assume that there exists $c_{1}>c_{2}$ such that $f_{c_{1}}^{\prime}(t) \rightarrow 0$ and $f_{c_{2}}^{\prime}(t) \rightarrow 0$ as $t \rightarrow+\infty$, and denote by $v_{1}$ and $v_{2}$ the functions associated to $f_{c_{1}}$ and $f_{c_{2}}$ by (4.1). If we set $w=v_{1}-v_{2}$ then $w\left(b^{2}\right)=0$ and $w^{\prime}\left(b^{2}\right)<0$. We claim that $w^{\prime}<0$ on $\left.] 0, b^{2}\right]$. For contradiction, assume there exists $\left.x \in\right] 0, b^{2}\left[\right.$ such that $w^{\prime}<0$ on ]0, $x\left[\right.$ and $w^{\prime}(x)=0$. Hence we have $w^{\prime \prime}(x) \leq 0$ and $w(x)>0$. But thanks to (4.1), we have

$$
w^{\prime \prime}(x)=\frac{w(x)}{\sqrt{x}} v_{1}^{\prime^{2}}(x)
$$

and a contradiction.

Now, let us set $V_{i}=\frac{1}{v_{i}^{\prime}}$ for $i=1,2$ and $W=V_{1}-V_{2}$. Then $W\left(b^{2}\right)=2\left(c_{1}-c_{2}\right)>0$ and $W(y) \rightarrow 0$ as $y \rightarrow 0$. In the other hand, thanks to (4.2); we have

$$
\left.\forall y \in] 0, b^{2}\right], \quad W^{\prime}(y)=-\frac{w(y)}{\sqrt{y}}-2 \beta \frac{y-1}{\sqrt{y}} w^{\prime}(y)
$$

Therefore, we have

$$
\begin{aligned}
W\left(b^{2}\right) & =\int_{0}^{b^{2}} W^{\prime}(y) d y \\
& =-\int_{0}^{b^{2}}\left(\frac{w(y)}{\sqrt{y}}+2 \beta \frac{y-1}{\sqrt{y}} w^{\prime}(y)\right) d y \\
& =-2[\sqrt{y} w(y)]_{0}^{b^{2}}+2 \int_{0}^{b^{2}}\left((1-\beta) \sqrt{y}+\frac{2 \beta}{\sqrt{y}}\right) w^{\prime}(y) d y \\
& =2 \int_{0}^{b^{2}}\left((1-\beta) \sqrt{y}+\frac{2 \beta}{\sqrt{y}}\right) w^{\prime}(y) d y,
\end{aligned}
$$

the last equality following from the fact that $w(y)$ tends to finite limit as $y \rightarrow 0$. Since $w^{\prime}<0$, we finally obtain $W\left(b^{2}\right)<0$ and a contradiction.

Remark 4.4. The change of variable (4.1) is particularly efficient to obtain some uniqueness results. In [4], it is used for the general equation (1.2) (cf. Section 4, lemma 5.4 and lemma 5.17). The case we examined in the previous proposition is part of lemma 5.17 of [4] with $\lambda=-1$.

Corollary 4.1. One has $\left.C_{2.2}=\right]-\infty, c_{*}\left[\right.$ and $C_{2.1}=\left[c_{*}, c^{*}[\right.$.

Proof. From remark 4.2, proposition 4.4 and 4.5, we see that $C_{2.2}$ is open, contains $]-\infty, c_{*}[$. Therefore, since $c_{*}=\inf \left(C_{1} \cup C_{2,1}\right)$, we necessarily have $\left.C_{2.2}=\right]-\infty, c_{*}\left[\right.$ and $C_{2.1}=\left[c_{*}, c^{*}[\right.$. 
To finish this section, let us express the results of proposition 4.1, proposition 4.2 and corollary 4.1 in terms of the boundary problems $\left(\mathcal{P}_{\beta} ; a, b,-1\right)$ and $\left(\mathcal{P}_{\beta ; a, b, 1}\right)$.

Theorem 4.1. Let $\left.\beta \in] 0, \frac{1}{2}\right], a \geq 0$ and $b \geq 1$. There exists $c_{*}<0$ such that:

(1) $f_{c}$ is not defined on the whole interval $\left[0,+\infty\left[\right.\right.$ if $c<c_{*}$;

(2) $f_{c_{*}}$ is a concave solution of $\left(\mathcal{P}_{\beta} ; a, b,-1\right)$;

(3) $f_{c}$ is a solution of $\left(\mathcal{P}_{\beta ; a}, b, 1\right)$ for all $\left.c \in\right] c_{*},+\infty[$

Moreover, there exists $\left.\left.c^{*} \in\right] c_{*}, \quad-a(b-1)\right]$;

(1) $f_{c}$ is a convex-concave solution of $\left(\mathcal{P}_{\beta ; a, b, 1}\right)$ for all $\left.c \in\right] 0,+\infty[$;

(2) $f_{c}$ is a concave solution of $\left(\mathcal{P}_{\beta ; a, b, 1}\right)$ for all $c \in\left[c^{*}, 0\right]$;

(3) $f_{c}$ is a concave-convex solution of $\left(\mathcal{P}_{\beta ; a, b, 1}\right)$ for all $\left.c \in\right] c_{*}, c^{*}[$;

5. The CASE $\left.\beta \in] 0, \frac{1}{2}\right]$ AND $-1<b<1$

Let $\left.\beta \in] 0, \frac{1}{2}\right], a \geq 0$ and $-1<b<1$. In this situation, it is easy to see that $\mathbb{R}$ can be partitioned into the four sets $C_{0,1}^{\prime}, C_{0,2}^{\prime}, C_{1}^{\prime}$ and $C_{2}^{\prime}$ where

$$
\begin{gathered}
C_{0,1}^{\prime}=\left\{c < 0 : f _ { c } ^ { \prime } > - 1 \text { on } \left[0, T_{c}[\},\right.\right. \\
C_{0,2}^{\prime}=\left\{c<0: \exists s_{c} \in\right] 0, T_{c}\left[\text { such that } f _ { c } ^ { \prime } > - 1 \text { on } \left[0, s_{c}\left[\text { and } f_{c}^{\prime}\left(s_{c}\right)=-1\right\},\right.\right. \\
C_{1}^{\prime}=\left\{c \geq 0 ; b \leq f _ { c } ^ { \prime } \leq 1 \text { and } f _ { c } ^ { \prime \prime } \geq 0 \text { on } \left[0, T_{c}[\},\right.\right. \\
C_{2}^{\prime}=\left\{\begin{array}{c}
c \geq 0 ; \exists t_{c} \in\left[0, T_{c}\left[, \quad \exists \epsilon_{c}>0 \text { such that } f_{c}^{\prime}<1 \text { on }\right] 0, t_{c}[,\right. \\
\left.f_{c}^{\prime}>1 \text { on }\right] t_{c}, t_{c}+\epsilon_{c}\left[\text { and } f_{c}^{\prime \prime}>0 \text { on }\right] 0, t_{c}+\epsilon_{c}[
\end{array}\right\} .
\end{gathered}
$$

The arguments used in the previous section, can be applied here.

First, since $g(x)=\beta\left(x^{2}-1\right)<0$ for $\left.\left.x \in\right]-1, b\right]$ where $\left.b \in\right]-1,0[$, the function $g$ is nonincreasing on ]$-1, b]$, it follows from theorem 5.5 of [4] that there exists a unique $c_{*}$ such that $f_{c_{*}}$ is a concave solution of $\left(\mathcal{P}_{\beta ; a, b,-1}\right)$. Moreover, we have $c_{*}<0$. As in the previous section, this implies that $\left.C_{0,2}^{\prime}=\right]-\infty, c_{*}[$. Hence $C_{0,1}^{\prime}=\left[c_{*}, 0[\right.$, and if $c \in] c_{*}, 0\left[\right.$, then $f_{c}^{\prime \prime}$ vanishes at a first point where $f_{c}^{\prime}<1$.

Next, in the same way as in the proof of proposition 3.1, we can proof that $c^{*}=\sup C_{1}^{\prime}$ is finite, and hence that $C_{1}^{\prime}=\left[0, c^{*}\right]$ and $\left.C_{2}^{\prime}=\right] c^{*},+\infty\left[\right.$. Moreover, from lemma 2.7, we have $c^{*} \geq a(1-b)$. On the other hand, it follows from lemma 2.6 that, if $c \in C_{2}^{\prime}$, then $f_{c}^{\prime \prime}$ vanishes at a first point where $f_{c}^{\prime}>1$.

All this, combined with an appropriate use of lemmas 2.7 and 2.8 allows to state the following theorem. 
Theorem 5.1. Let $\left.\beta \in] 0, \frac{1}{2}\right]$ and $-1<b<1$. There exist $c_{*}<0$ and $c^{*} \geq a(1-b)$ such that:

(1) $f_{c}$ is not defined on the whole interval $\left[0,+\infty\left[\right.\right.$ if $c<c_{*}$;

(2) $f_{c_{*}}$ is a concave solution of $\left(\mathcal{P}_{\beta} ; a, b,-1\right)$ if $\left.b \in\right]-1,0[$;

(3) $f_{c}$ is a concave-convex solution of $\left(\mathcal{P}_{\beta} ; a, b, 1\right)$ for all $\left.c \in\right] c_{*}, 0[$;

(4) $f_{c}$ is a convex solution of $\left(\mathcal{P}_{\beta} ; a, b, 1\right)$ for all $c \in\left[0, c^{*}\right]$;

(5) $f_{c}$ is a convex-concave solution of $\left(\mathcal{P}_{\beta ; a, b, 1}\right)$ for all $\left.c \in\right] c^{*},+\infty[$.

Conflicts of Interest: The author(s) declare that there are no conflicts of interest regarding the publication of this paper.

\section{REFERENCES}

[1] M. Aiboudi and B. Brighi, On the solutions of a boundary value problem arising in free convection with prescribed heat ux, Arch. Math. 93(2), 2009, 165-174.

[2] M. Aiboudi, K. Boudjema Djeffal, and B. Brighi, On the Convex and Convex-Concave Solutions of Opposing Mixed Convection Boundary Layer Flow in a Porous Medium, Abstr. Appl. Anal. 2018 (2018), Article ID 4340204, 5 pages.

[3] M. Aiboudi, I. Bensari and B. Brighi, Similarity solutions of mixed convection boundary-layer flows in a porous medium, Differ. Equ. Appl. 9(1)(2017), 69-85.

[4] B. Brighi, The equation $f^{\prime \prime \prime}+f f^{\prime \prime}+g\left(f^{\prime}\right)=0$ and the associated boundary value problems, Results Math. 61(3-4)(2012), 355-391.

[5] B. Brighi, A. Fruchard and T. Sari, On the Blasius problem, Adv. Differ. Equ, 13(5-6), 2008, 509-600.

[6] B. Brighi and J.D. Hoernel, On similarity solutions for boundary layer ows with prescribed heat flux, Math. Methods Appl. Sci. 28(4)(2005), 479-503.

[7] B. Brighi and J.D. Hoernel, On the concave and convex solutions of mixed convection boundary layer approximation in a porous medium, Appl. Math. Lett. 19(2006), 69-74.

[8] B. Brighi and J-D. Hoernel, On a general similarity boundary layer equation, Acta Math. Univ. Comenian. 77(1)(2008), $9-22$.

[9] D. R. Hartree, On an equation occurring in Falkner and Skan's approximate treatment of the equations of the boundary layer, Math. Proc. Cambridge Phil. Soc. 33(2)(1937), 223-239.

[10] G.C. Yang, A note on $f^{\prime \prime \prime}+f f^{\prime \prime}+\beta\left(f^{\prime 2}-1\right)=0$ with $\lambda \in\left(-\frac{1}{2}, 0\right)$ arising in boundary layer theory, Appl. Math. Lett. $17(2004), 1261-1265$.

[11] G.C. Yang and J. Li, A new result on $f^{\prime \prime \prime}+f f^{\prime \prime}+\beta\left(f^{\prime 2}-1\right)=0$ arising in boundary layer theory, Nonlinear Funct. Anal. Appl. 10(2005), 117-122.

[12] G.C. Yang, L.L. Shi and K.Q. Lan, Properties of positive solutions of the Falkner-Skan equation arising in boundary layer theory, in: Integral Methods in Science and Engineering, Birkhäuser, Boston, Boston, MA, 2008, 277-283.

[13] G.C. Yang, Existence of solutions of laminar boundary layer equations with decelerating external flows, Nonlinear Anal., Theory Methods Appl. 72(2010), 2063-2075.

[14] G.C.Yang, An extension result of the opposing mixed convection problem arising in boundary layer theory, Appl. Math. Lett. 38(1)(2014), 180-185.

[15] H. Weyl, On the Differential Equations of the Simplest Boundary-Layer Problems, Ann. Math. 43(2)(1942), 381-407. 
[16] W. A. Coppel, On a Differential Equation of Boundary-Layer Theory, Phil. Trans. R. Soc. Lond. Ser. A, Math. Phys. Eng. Sci. 253(1023)(1960), 101-136.

[17] P. Hartman, Ordinary Differential Equations, JohnWiley \& Sons, New York, NY, USA, 1964. 